\title{
Le choix des médecins experts
}

Jean-François Boudry,

Jean-Paul Studer, Gilbert Villard, Joël Rilliot

Les médecins traitants déplorent souvent l'attitude de leurs confrères effectuant des expertises. Ils la ressentent parfois comme un abus de pouvoir perpétré à l'égard de leurs patients. Lors de réunion entre eux, ils échangent fréquemment leurs plaintes. Ne serait-il pas temps de réagir collectivement? De susciter une réflexion permettant d'apporter des améliorations à une situation problématique?

L'avis du médecin traitant, celui qui suit un patient depuis des années, est de moins en moins pris en compte. Certes, on le sollicite encore, mais vraisemblablement pour la forme, car, le plus souvent, son point de vue et ses conclusions ne sont

\section{L'avis du médecin traitant, celui qui suit un patient depuis des années, est de moins en moins pris en compte. Certes, on le sollicite encore, mais vraisemblablement pour la forme}

Correspondance:

Dr Jean-François Boudry Grand-Rue 4

CH-2000 Neuchâtel

jfboudry@bluewin.ch guère retenus. Est-il considéré comme suspect, partial, non objectif, privilégiant son patient? Je ne vois guère d'autres explications au peu de cas qui est fait en général de ses rapports et certificats. On lui préfère l'avis d'un médecin qui, sur la base d'un entretien ponctuel, certes parfois assorti d'examens complémentaires et/ou pluridisciplinaires, statue de façon péremptoire et définitive, apparemment sans le moindre doute ni le moindre état d'âme, et le plus souvent, il faut le dire, dans un sens favorable à l'assureur (assureur maladie, accident ou invalidité).

Si le médecin traitant se permet d'émettre des doutes quant à l'objectivité d'un expert, ses propos sont taxés d'atteinte grave à la déontologie ... La non-prise en compte de son avis, le mépris fréquent avec lequel il est jugé, ne sont-ils pas aussi des atteintes à la déontologie? Certes, au fil des années, le médecin traitant noue une relation privilégiée avec ses patients, mais le taxer systématiquement de partial, c'est considérer qu'il fait preuve de légèreté et d'inconscience vis-à-vis des institutions sociales, donc douter de son éthique professionnelle. C'est pour le moins une accusa- tion grave. Le médecin expert, n'est-il pas lié aux instances qui le sollicitent? Dont il reçoit travail et rémunération? De quel droit lui réserver à lui seul le label d'objectivité?

Le recours à une expertise se justifie bien sûr dans des cas litigieux. Force est de constater qu'actuellement le doute profite aux assureurs. Les arguments du médecin traitant sont le plus souvent balayés, non pris en compte. La situation économique, les comptes déficitaires des assurances sociales, mais aussi, pour les assurances privées, la dictature du profit maximum expliquent cet état de fait. Certains questionnaires peuvent être tendancieux. Parfois le médecin expert appuie son argumentation sur un entretien téléphonique qu'il a eu avec le médecin traitant. Malheureusement il n'est pas rare que les propos échangés à cette occasion soient interprétés dans le sens souhaité par l'expert. Le médecin traitant ne devrait plus accepter de répondre téléphoniquement et exiger des questions écrites auxquelles il aurait droit d'apporter corrections, commentaires et remarques.

Le médecin expert porte souvent sur les avis du médecin traitant des jugements péremptoires et définitifs, teintés parfois de suffisance. Il oublie alors le long suivi d'un patient dans la durée, avec tout l'arrière-fond existentiel, la somme de doutes et d'incertitudes qui jalonne fréquemment l'histoire de ces malades à problème assécurologique. Il tranche apparemment sans nuance, sûr de son avis. A-t-il conscience du risque d'un éventuel abus de pouvoir?

\section{Le recours à une expertise se justifie bien sûr dans des cas} litigieux. Force est de constater qu'actuellement le doute profite

\section{aux assureurs}

Combien de patients, maintenus en équilibre précaire ou en lente voie d'amélioration, régressent ou s'effondrent après une expertise? Cette «épreuve» est ressentie par eux comme froide et 
impersonnelle. Ils la vivent comme s'ils étaient suspectés d'être des simulateurs cherchant à abuser la société.

La médecine n'est pas une science exacte. Quotidiennement le médecin traitant, par ses choix diagnostiques et thérapeutiques, s'expose à l'épreuve du temps. L'avenir lui dira, lorsqu'il reverra son patient, si ses options ont été judicieuses. Au besoin, il les adaptera, les modifiera, se remettra en question. La médecine est avant

\section{Selon quels critères les médecins experts sont-ils choisis? Nous estimons quant à nous que la société médicale du canton concerné devrait être consultée sur ce choix}

1 Victor Yves Ghebali, professeur honoraire à la Haute Ecole d'Etudes Internationales et du Développement à Genève, dans le journal «Le Temps» du 28 octobre 2008. tout et surtout une école d'humilité et de modestie. Le médecin expert ne voit le patient qu'une fois. On lui demande de trancher, d'affirmer, de statuer. Il le fait sur la base de ce seul contact dont l'importance nous semble excessive. Il s'appuie certainement aussi sur le dossier médical, mais sa lecture peut bien sûr donner lieu à différentes interprétations. Sans mettre en doute l'honnêteté de l'expert, nous avons le sentiment que l'impression qu'il a du patient primera sur toute autre considération. En tant que médecin, il doit pour- tant savoir que l'état d'un malade est le plus souvent fluctuant au fil du temps. Et qu'une expertise est une épreuve particulière pour lui. A laquelle il réagira avec plus ou moins de naturel, de blocages, d'anxiété, d'agressivité. Et c'est dans ce contexte que l'avis de l'expert a force de loi. C'est une médecine particulière: elle donne l'illusion de répondre aux critères d'une science exacte! Avec des répercussions psychologiques et sociales que semble ignorer ou banaliser l'expert, mais que mesure le médecin traitant.

Selon quels critères les médecins experts sontils choisis? Nous estimons quant à nous que la société médicale du canton concerné devrait être consultée sur ce choix. De même que le médecin traitant. Pour qu'ensemble ils établissent le cahier des charges. En outre, pour une plus grande objectivité dans l'évaluation globale de la problématique d'un patient, il nous paraîtrait judicieux que l'expert choisi soit de la même spécialité que le médecin traitant. Enfin, les conclusions de l'expert devraient être transmises en priorité au médecin traitant afin qu'il ait le droit de les discuter avant que la décision ne soit communiquée au patient. Le tout dans un climat de profond respect mutuel.

Et pour terminer, une citation en guise de clin d'œil: «Un expert, n'est-il pas quelqu'un qui se trompe avec plus de compétence que les autres?» [1] 